\title{
Cardiac endothelial cells isolated from mouse heart - a novel model for radiobiology
}

\author{
Karol Jelonek ${ }^{1}$, Anna Walaszczyk1', Dorota Gabryś1, Monika Pietrowska1', Chryso Kanthou² \\ and Piotr Widłak ${ }^{1 / 2}$
}

\begin{abstract}
Cardiovascular disease is recognized as an important clinical problem in radiotherapy and radiation protection. However, only few radiobiological models relevant for assessment of cardiotoxic effects of ionizing radiation are available. Here we describe the isolation of mouse primary cardiac endothelial cells, a possible target for cardiotoxic effects of radiation. Cells isolated from hearts of juvenile mice were cultured and irradiated in vitro. In addition, cells isolated from hearts of locally irradiated adult animals (up to 6 days after irradiation) were tested. A dose-dependent formation of histone $\mathrm{\gamma H} 2 \mathrm{~A} . \mathrm{X}$ foci was observed after in vitro irradiation of cultured cells. However, such cells were resistant to radiation-induced apoptosis. Increased levels of actin stress fibres were observed in the cytoplasm of cardiac endothelial cells irradiated in vitro or isolated from irradiated animals. A high dose of 16 Gy did not increase permeability to Dextran in monolayers formed by endothelial cells. Up-regulated expression of Vcam 1, Sele and Hsp70i genes was detected after irradiation in vitro and in cells isolated few days after irradiation in vivo. The increased level of actin stress fibres and enhanced expression of stress-response genes in irradiated endothelial cells are potentially involved in cardiotoxic effects of ionizing radiation.
\end{abstract}

Keywords: cardiac endothelial cells, cardiotoxicity, primary cell culture, radiation, stress response

Received: 02 April, 2011; revised: 01 July, 2011; accepted: 01 August, 2011; available on-line: 31 August, 2011

\section{INTRODUCTION}

High radio-tolerance of the heart was for a long time a general paradigm of radiobiology and radio-oncology. The tolerance dose of the heart in clinical radiotherapy has been estimated to about $40 \mathrm{~Gy}$ in whole organ irradiation (review in: Schultz-Hector \& Trott, 2007). However, an increased rate of heart failure observed in several groups of oncological patients treated with radiotherapy, such as Hodgkin's disease or breast cancer patients, initiated re-evaluation of the potential risk related to heart irradiation (Hancock et al., 1993; SenkusKonefka \& Jassem, 2007; Hendry et al., 2008). The risk of cardiovascular disease associated with radiotherapy is now recognized as an important clinical problem, often manifesting decades after the initial exposure (Little et al., 2008; Darby et al., 2010). Moderate to low doses delivered to the heart may also pose a long term risk as recently established by a large epidemiological study on atomic bomb survivors who received 0.5 to 2 Gy total body irradiation (Shimizu et al., 2010). Unfortunately, very few relevant radiobiological models are available at present for the assessment of heart radio-toxicity that consider the complex and long-term nature of radiotherapy-related damage involved in heart failure.

Among different heart structures critical for radiationinduced damage the heart microvasculature is an obvious candidate even though endothelial cells comprise only a minor fraction of cardiac cells. The endothelium forms a continuous monolayer of cells with barrier properties, which regulate the passage of plasma proteins, macromolecules and circulating cells between the blood and the interstitial tissues and is essential for vascular homeostasis (review in: Petty \& Pearson, 1989; Celermajer, 1997). Several studies have demonstrated that high doses of ionizing radiation induce immediate cellular damage to the vascular endothelium, resulting in the exposure of basal lamina and leakage of plasma proteins into the interstitial space. A rise in vascular permeability after exposure to radiation is generally considered as a crucial element in the development of radiotherapy-associated complications of edema and subsequent fibrosis (Hinsbergh, 1997; Gabrys et al., 2007). The exposure of endothelial cells to ionizing radiation in vitro affected the structure of the cytoskeleton, organization of actin microfilaments and intercellular junctions; however, the observed effects were dose- and cell origin-dependent (Kantak et al., 1993; Waters et al., 1996; Gabrys et al., 2007). We previously analyzed changes induced in vitro by ionizing radiation in the actin cytoskeleton of primary endothelial cells delivered from human dermal microvasculature (DMEC) and large vessels (HUVEC), and found that DMEC but not HUVEC cells respond to radiation by a rapid and dose-dependent formation of so called stress fibres (Gabrys et al., 2007). Endothelial cells are indeed very heterogeneous and display distinct and specialized properties in the vasculature of different organs. Large and small vessels respond differently to irradiation, and radiation-induced damage could be more severe in cells from the microvasculature (Gabrys et al., 2007). For

e-mail: widlak@io.gliwice.pl

Abbreviations: CEC, cardiac endothelial cells; DAPI, 4,6-diamidino-2-phenylindole; DMEC, dermal microvascular endothelial cells; DMEM, Dulbecco's Modified Eagle's Medium; DSB, DNA doublestrand break; Sel-E, E-selectin cell adhesion protein; FBS, fetal bovine serum; FITC, fluorescein isothiocyanate; Hsp, heat shock protein; HUVEC, human umbilical vein endothelial cells; Pecam-1, platelet endothelial cell adhesion molecule 1 (also termed CD31, endothelial-specific cell surface antigen); SA $\beta$-Gal, senescence-associated $\beta$-galactosidase; Vcam-1, vascular cell adhesion protein 1 
these reasons the commonly used endothelial cells isolated from aorta, umbilical vein or lymph nodes are not the most relevant models to study radiation-induced malfunction of the heart microvasculature.

Here we aimed to optimize primary endothelial cell isolation form hearts of mice, either control or previously irradiated in vivo, and used these isolated cells for further culturing and assessment of stress responses to ionizing radiation.

\section{MATERIALS AND METHODS}

Animals and irradiation. Male C57/BL6 mice were used for experiments. Eight-week old animals were irradiated with $6 \mathrm{MeV}$ photons under Avertin anaesthesia using a linear accelerator (Clinac 600) with 2 or 8 Gy doses delivered to the whole heart volume, and then sacrificed at different times after irradiation (from 12 hours to 6 days). Overall, for each experimental point cells were purified from hearts of 4-6 animals irradiated in vivo. All procedures involving animals and their care were conducted in conformity with institutional guidelines in compliance with national and international laws and policies; the study was approved by the appropriate Ethical Committee.

Isolation of cardiac endothelial cells (CEC). CEC were isolated from C57/BL6 mice. Collected hearts (proportions indicated below were standardized for isolation from two hearts of adult mice or four hearts of juvenile mice) were rinsed intensively with phosphatebuffered saline (PBS) to remove blood and then minced into small pieces with a sterile scalpel blade. The tissue fragments were transferred to $10 \mathrm{ml}$ of solution that consisted of DMEM containing $10 \%$ FBS, $0.16 \mathrm{mg} /$ $\mathrm{ml}$ gentamycin, $4 \mathrm{mM}$ L-glutamine and 0.3 units $/ \mathrm{ml}$ of collagenase A (Roche; cat. no. 10.103.586.001), and incubated at $37^{\circ} \mathrm{C}$ for $45-60$ minutes with continuous shaking. Any remaining clumps of cells were dispersed by forcing through a sterile $18 \mathrm{G}$ needle 20 times. The resulting mixture was filtered through $30-\mu \mathrm{m}$ pore-size pre-separation filter (MACS Miltenyi Biotec) and the single-cell suspension was washed twice in DMEM with centrifugation at $400 \times \mathrm{g}$ for 6 minutes after which collected cells were counted. The final cell pellet consisting of about 40-50 million cells was re-suspended in 6 $\mathrm{ml}$ of DMEM. A suspension of $8 \mu \mathrm{l}$ of sheep anti-rat Dynabeads (Invitrogen; cat. no. 110.35), which were precoated with rat anti-mouse CD31 (also termed Pecam-1) antibody (Pharmingen; cat. no. 553370) according to manufacturer's instructions, was added to the cell suspension; this corresponded to approximately one Dynabead particle per 20 cells. The mixture was incubated for 20 minutes at room temperature in a non-adhering $15 \mathrm{ml}$ tube with gentle agitation. The unbound cells were removed from the mixture after Dynabead-bound cells were immobilized on the tube wall using a magnet (DynaMag-15, Invitrogen). Bound cells were washed 5 times with $6 \mathrm{ml}$ DMEM using the same magnet device (each time cells were vortexed for 5 seconds after adding fresh medium). Immuno-selected CD31-positive cells were counted and plated on Petri dishes or Permanox Lab-Tek 4-well chamber slides with cover lids (Nunc) in EBM-2 basal endothelial cell medium (Lonza; cat. no. CC3156) supplemented with EGM-2 growth factor mixture (Lonza; cat. no. CC4176); initial concentration of cells was approximately $2.5 \times 10^{4}$ per $\mathrm{cm}^{2}$. Cells were cultured in standard conditions $\left(37^{\circ} \mathrm{C}, 5 \% \mathrm{CO}_{2}\right)$. Next day, the cultures were washed gently with the same medium to remove unattached cells (which were counted to estimate proportion of adherent and non-adherent cells) and excess of Dynabeads; thereafter the medium was changed every two days. Cells were counted again once they reached confluence around 5-9 days after the initial isolation depending on the age of heart donors. CECs were considered to be confluent after covering at least $80 \%$ of the well area. For some experiments, cells were irradiated in vitro at this point.

Staining for endothelial cell surface markers, actin cytoskeleton and $\beta$-galactosidase. Confluent cultures were fixed for 15 minutes in $10 \%$ formaldehyde. Cell surface marker CD31 was detected using purified rat anti-mouse CD31 antibody (Pharmingen; at a 1:100 dilution). Cells were incubated with the primary antibody diluted in 2\% BSA and 5\% normal horse serum in PBS at $4{ }^{\circ} \mathrm{C}$ overnight, and then with a fluorescein-conjugated anti-rat Ig antibody (Vector; at a 1:100 dilution) for 1 hour at room temperature. Actin structures were labelled with Texas-Red-conjugated phalloidin (Molecular Probes; at a 1:200 dilution); incubation was performed in $10 \mathrm{mM}$ Hepes, $\mathrm{pH} 7.5$ for $2 \mathrm{~h}$ at room temperature in the dark. To visualize nuclei, slides were stained with DAPI-containing Vectashield mounting medium (Vector Laboratories). Fluorescence images were captured with a Nikon Eclipse 80i inverted microscope. For each experimental point (with 4 technical repeats) actin fibres were evaluated in 50 randomly chosen cells; cells were divided into three groups differing in the number of visible actin stress fibres classified as $0,1-10$ and $>10$. For $\beta$-galactosidase staining PBS-washed cells were fixed for 3 minutes in $3 \%$ formaldehyde, washed and incubated for 6 hours at $37^{\circ} \mathrm{C}$ in freshly prepared staining solution containing $0.1 \% \mathrm{X}-\mathrm{Gal}$ in $40 \mathrm{mM}$ citric acid/sodium phosphate, $\mathrm{pH} 6.0,5 \mathrm{mM}$ potassium ferrocyanide, $5 \mathrm{mM}$ potassium ferricyanide, $150 \mathrm{mM} \mathrm{NaCl}$ and $2 \mathrm{mM} \mathrm{MgCl}_{2}$.

Detection of $\gamma \mathrm{H} 2 \mathrm{~A} . \mathrm{X}$ foci and TUNEL assay. CECs cultured in Permanox Lab-Tek 4-well chamber slides were washed twice for 2 minutes with PBS and then fixed for 30 minutes with $10 \%$ formaldehyde; repeated washes with PBS were applied after each further step of the procedure. Fixed cells were incubated for 4 minutes with $0.1 \%$ Triton in $0.1 \times$ SSC for membrane permeabilization, and then for 30 minutes with $3 \%$ BSA in PBS for non-specific blocking. Cells were incubated for one hour at room temperature with mouse antiphospho-histone H2A.X monoclonal antibody (Millipore; cat. no. 05-636) in 3\% BSA/PBS (at a 1:250 dilution), and then for one hour at room temperature with antimouse IgG biotinylated antibody (Vector; cat. no. BA1400 ) in $3 \%$ BSA/PBS (at a 1:250 dilution). To visualize the $\gamma \mathrm{H} 2 \mathrm{~A}$.X foci cells were incubated in the dark for 40 minutes at room temperature with Texas-Red-conjugated streptavidin (Vector; cat. no. SA-5006) in $10 \mathrm{mM}$ Hepes $\mathrm{pH} 7.5$ (at a 1:250 dilution). To visualize nuclei cells were incubated in the dark for 10 minutes at room temperature with $300 \mathrm{nM}$ DAPI, and then fluorescence images were captured with a Nikon Eclipse 80i inverted microscope. The number of $\gamma \mathrm{H} 2 \mathrm{~A}$.X foci per nucleus was counted for at least one hundred randomly selected cells. To assess for apoptosis using the TUNEL assay, cells were fixed with $4 \%$ formaldehyde for 30 minutes at room temperature, washed with $\mathrm{PBS}$ and permeabilized with $0.1 \%$ Trion X-100 in $0.1 \%$ sodium citrate for 4 minutes on ice. After washing with PBS $50 \mu$ of the TUNEL reaction mixture (Fluorescein In Situ Cell Death Detection Kit, Roche) was added, then samples were 
covered with parafilm and incubated in a humidified atmosphere for 90 minutes at $37^{\circ} \mathrm{C}$ in the dark. Samples were then washed, counterstained with DAPI and analyzed under a fluorescence microscope.

Measurement of endothelial permeability. Cells were plated on $6-\mathrm{mm}$ diameter $0.3 \mu \mathrm{m}$ pore-size filters (Falcon) pre-coated with $10 \mu \mathrm{g} / \mathrm{ml}$ fibronectin (Invitrogen). A total of $10^{5}$ cells were plated per filter and set into a 24-well companion plate, and then $200 \mu \mathrm{l}$ of the growth factor-supplemented EBM-2 medium in the insert/upper chamber and $700 \mu \mathrm{l}$ of medium in the lower chamber was used (medium was changed every other day after the plating). The integrity of the cell monolayer was confirmed by staining of additional filters with Texas-Red-conjugated phalloidin and DAPI. Cells in the monolayer were irradiated on filters with a 16 Gy dose, and then 3 or 24 hours after irradiation the medium in the upper chamber was replaced with medium supplemented with $0.8 \mathrm{mg} / \mathrm{ml}$ of FITC-Dextran, MW 40000 (Sigma). After one hour of incubation $100 \mu$ l of medium was collected from the lower compartment (for measurement the sample was diluted 10 -fold with water). The fluorescence was measured using a Fluorolog 3-12 fluorimeter (Jobin von Spex) at $488 \mathrm{~nm}$ excitation and 525 $\mathrm{nm}$ emission wavelengths. Cultures pre-incubated for 3 hours with $5 \mathrm{mM}$ EGTA, which disrupted integrity of inter-cellular junctions (Ponnuchamy \& Khalil, 2008), were used as positive controls of the assay.

Gene expression analysis. Collected cells (either directly after immuno-selection or cultured and harvested from culture dishes) were washed in PBS, dissolved in Trizol, and then stored at $-70^{\circ} \mathrm{C}$. Total RNA was extracted from frozen material using a Total RNA Isolation Kit (A\&A Biotechnology) according to the manufacturer's protocol. The cDNA synthesis was performed using a Revertaid First Strand cDNA Synthesis Kit (Fermentas) according to the manufacturer's protocol using $1 \mu \mathrm{g}$ of RNA templates. PCR reaction mixtures contained $25 \mathrm{ng}$ of cDNA, 25 pmoles of each starter, $1 \mathrm{U}$ of Taq polymerase, $2 \mathrm{mM}$ of each dNTP, $25 \mathrm{mM}$ $\mathrm{MgCl}_{2}, 500 \mathrm{mM} \mathrm{KCl}$ and $100 \mathrm{mM}$ Tris/HCl, pH 8.3 in a total volume of $10 \mu \mathrm{l}$. Products specific for different transcripts were analyzed and the temperature for primer annealing was optimized for each reaction. Primers used in the analysis specifically amplified transcripts of the following genes: V cam1 (GenBank accession no. NM_011693.3; 288 bp fragment from position 1037 to 1324), Sele (NM_011345.2; 266 bp fragment from position 387 to 652), Bnip3 (NM_009760.3; 156 bp fragment from position 359 to 514), Glut1 (NM_011400.2; $210 \mathrm{bp}$ fragment from position 1248 to 1457$), H s p A 1 A$ / HspA1B (hereafter referred to as Hsp70i) (NM_010479.2; 699 bp fragment from position 38 to 736), Gapdh (XR_033802.1; 298 bp fragment from position 300 to 597). PCR products were separated electrophoretically on $1.5 \%$ agarose gels and visualized after staining with ethidium bromide. Three independent experiments were performed with cells irradiated in vitro and three technical repeats of assays with pooled cells isolated from 3 animals irradiated in vivo were performed, and then gels were analyzed by densitometry for quantification (Fig. 5 shows typical electrophorograms).

Statistical analysis. For each experimental point cells were purified from hearts of 2-4 animals, and then 3-5 technical repeats of the assay were performed. For some assays experiments were performed using 2-3 independent pools of cells (each with 3-5 technical replicas). Statistical analyses were performed assuming normal distribution of experimental data (which was verified using Kolmogorov-Smirnov and Shapiro-Wilk tests) using Statistica 9.1 software. Statistical significance was assessed using the unpaired two sample Student's t-test; $p$ value lower than 0.05 was considered as the significance cut-off level.

\section{RESULTS}

\section{Characterization of endothelial cells isolated from murine hearts}

To isolate primary endothelial cells from the heart we took advantage of the positive immunoselection strategy using endothelium-specific cell-surface antigens. To optimize conditions of the isolation procedure different types of cell separation/tissue dispersion methods (including collagenase and/or trypsin treatment), endothelial cell-specific antigens (CD31, CD102, CD105), magnetic beads and devices (Dynabeads vs. MACS System), and culture media were tested. Eventually, a procedure using collagenese treatment of the tissue followed by CD31 (Pecam-1)-based positive cell selection using the Invitrogen Dynabeads system allowed us to reach a high yield of pure endothelial cells from hearts of C57/BL6
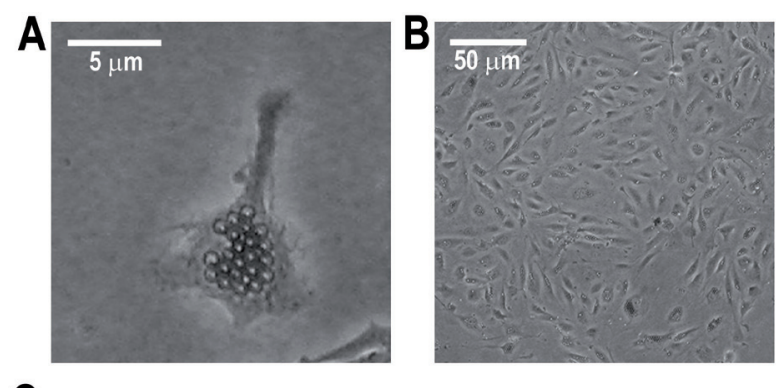

\section{C \\ D}
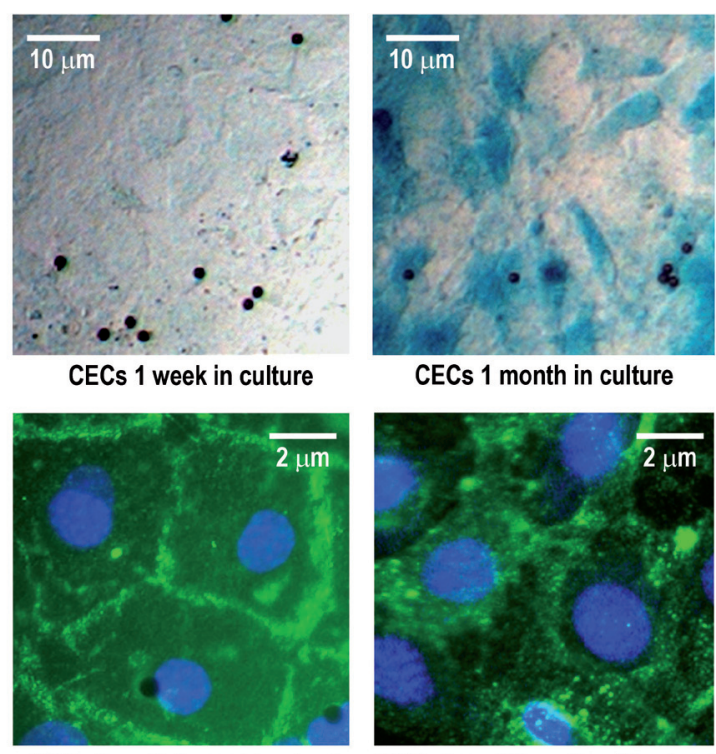

CECs from 3 days-old animal

Figure 1. Morphology of isolated cardiac endothelial cells

(A) A single CEC directly after immunoselection; magnetic beads attached to the cell are visible. (B) Confluent culture of CECs isolated from a 20-week-old animal one week after immunoselection. (C) CECs isolated from a juvenile animal were stained for senescence-associated $\beta$-galactosidase after one week or one month in culture; cells were observed in phase contrast without counterstaining. (D) CECs immuno-stained with anti-CD31 antibody (with DAPI counterstaining); shown are confluent cells isolated from 3-day-old and 28-week-old mice. 
mice. Figure 1 (panel A and B) shows microphotographs of isolated cardiac endothelial cells (CECs) immunoselected with anti-CD31-coated Dynabeads.

Cardiac endothelial cells were isolated from hearts of mice of different age, juvenile (1-5 days) or adult (up to 88 months) animals, to compare yield and the ability of CECs to grow in culture. Table 1 shows the number of endothelial cells counted directly after immunoselection and the number of adherent cells that grew on a gelatin-coated plastic surface one week after isolation. More cells were isolated from older animals, which likely corresponds to the larger size of their heart. However, the number of adherent cells growing in culture was higher for younger animals than for older ones. Importantly, substantial proliferation of endothelial cells in culture was found only for cells from juvenile hearts, whose number doubled during the first week of culture. This correlated with the ability of such cells to persist in culture for at least 5-6 weeks (3-4 passages) while cells isolated from adult animals (5-6 months old) could be cultured only for 2-3 weeks. Notably, endothelial cells isolated from hearts of elderly mice (about 11 months and older) have totally lost their ability to adhere and grow on the surface of a culture dish. Most importantly, when endothelial cells were isolated from hearts of irradiated mice 20 weeks after exposure to up to $8 \mathrm{~Gy}$ the number of cells and their ability to grow in vitro were similar to those of cells isolated from unirradiated mice of the same age (not shown). To search for a cause of the lost viability in culture, isolated CECs were tested for the activity of
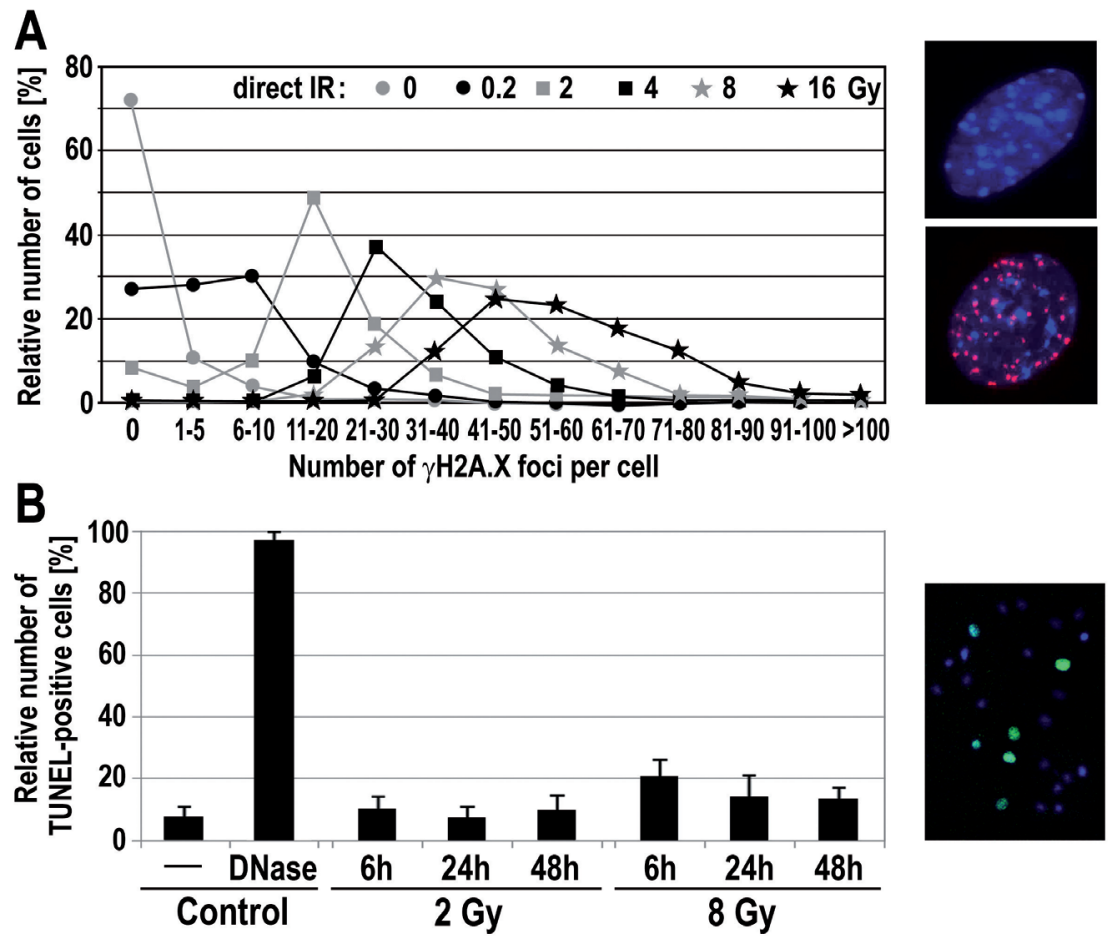

senescence-associated $\beta$-galactosidase (SA- $\beta$-Gal), a generally accepted marker of cells undergoing senescence (Dimri et al., 1995). CECs isolated from hearts of juvenile mice were stained for SA- $\beta-G a l$ after one week or one month in culture. No cells stained after one week in culture while almost all cells became SA- $\beta$-Gal-positive after one month in culture (Fig. 1C), which indicated induction of senescence in those cells. The morphology of cultured endothelial cells isolated from juvenile and adult animals was also compared. After one week in culture the cells were fixed and stained using anti-CD31 antibody (with DAPI counterstaining) to visualize their shape, boundaries and cell-to-cell contacts (Fig. 1D). We found that only cells isolated from juvenile animals could grow to form a monolayer and develop complete cell-tocell contacts. We concluded that endothelial cells isolated from hearts of juvenile mice were the best option for medium-term in vitro experiments which included irradiation of cells in culture.

\section{Radiation-induced DNA breaks and apoptosis}

DNA lesions are among the primary damage induced in cells exposed to ionizing radiation. DNA doublestrand breaks (DSBs) are generally considered to be the most toxic effects of radiation, and thus are commonly used in radiosensitivity tests (review in: Ward, 1988). We used immunocytochemistry-based assessment of nuclear foci of phosphorylated histone H2A.X, so called $\gamma$ H2A.X foci. Such foci form at sites of DSBs, which allows quantitative analysis of radiation-induced damage and its repair (Rogakou et al., 1998). Endothelial cells isolated from juvenile animals were irradiated in vitro with doses of up to 16 Gy. One hour after the exposure the cells were fixed and stained, and the number of $\gamma$ H2A.X foci per nucleus was determined. Figure $2 \mathrm{~A}$ shows percentage of cells with different levels of DSBs induced after exposure to increasing doses of ionizing radiation (from 0.2 to 16 Gy). The vast majority of control (non-irradiated) CECs contained no $\mathrm{DSB} / \gamma \mathrm{H} 2 \mathrm{~A}$.X foci while irradiation with the lowest dose $(0.2$ Gy) induced some DSB in the majority of cells. After irradiation with higher doses ( $\geq 2$ Gy) DSBs were detected in all cells; increasing doses led to increasing numbers of foci per nucleus. We concluded that, in respect to the formation of radiationinduced DSBs, isolated CECs showed a clear dose-dependent response after direct irradiation in vitro. To assess for radiationinduced apoptosis, CECs isolated from juvenile animals were irradiated in vitro with 2 or 8 Gy doses and then TUNEL-positive cells with apoptotic chromatin fragmentation were detected 6,24 and 48 hours after the exposure (Fig. 2B). We found that irradiation caused only a minor increase
Figure 2. Radiation-induced DNA damage and apoptosis of cardiac endothelial cells (A) Radiation-induced DNA double strand breaks detected as $\gamma \mathrm{H} 2 \mathrm{~A} . \mathrm{X}$ foci in nuclei of CECs 60 minutes after the exposure. Shown are mean values from 3 repeats of assay. Micrograph as red dots). (B) Assessment of cells with apoptotic chromatin fragmentation. Cells isolated from juvenile mice were exposed to 2 Gy or $8 \mathrm{~Gy}$, and then proportion of TUNEL-positive cells was determined 6, 24 and 48 hours after exposure (non-irradiated cells treated with DNase I were used as a positive control). Shown are mean values from 3 repeats of assay $( \pm$ S.D.). Micrograph on the right shows an example of TUNEL-positive (green) and TUNELnegative (blue) cells. 60 minu 


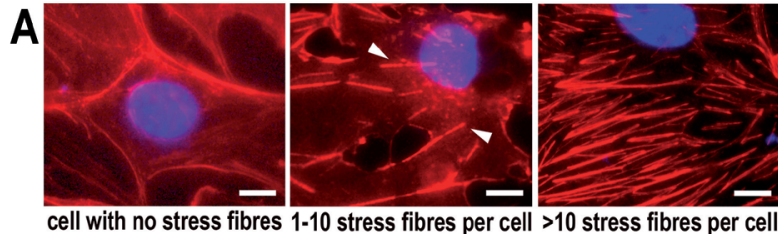

B
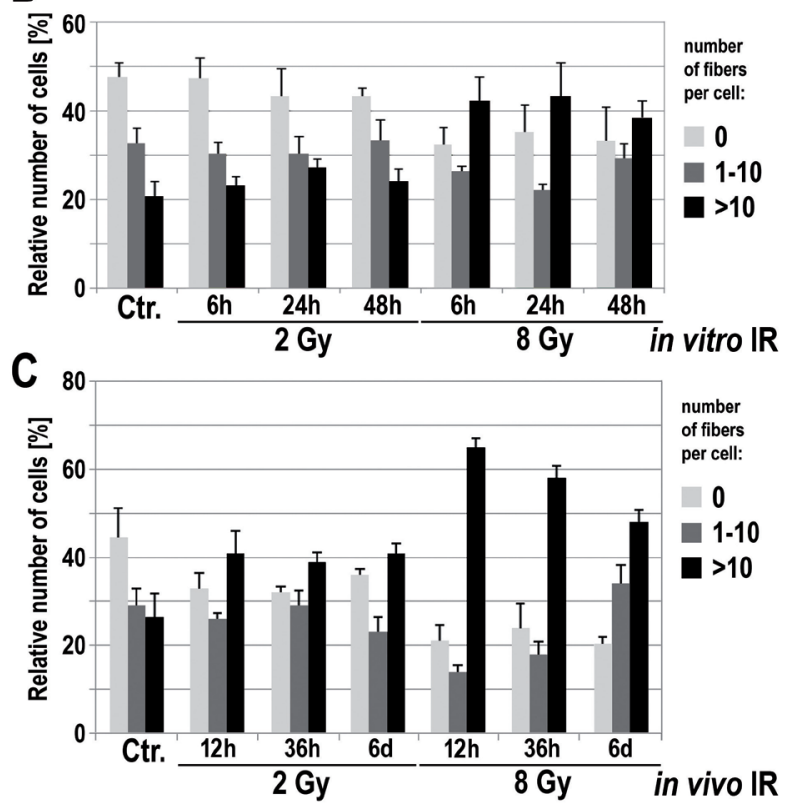

Figure 3. Radiation-induced stress fibers in cardiac endothelial cells

(A) Cells with different numbers of actin stress fibers (marked with arrowheads); nuclei stained with DAPI, scale bar $-1 \mu \mathrm{m}$. (B) CECs isolated from juvenile mice were exposed in vitro to 2 or $8 \mathrm{~Gy}$, and then actin cytoskeleton was visualized using fluorescence-labelled phalloidin 6, 24 and 48 hours after irradiation; diagram shows percentage of cells with different numbers of stress fibers. (C) CECs were isolated from 8-week-old mice 12 and 36 hours, and 6 days after animal irradiation with 2 or $8 \mathrm{~Gy}$, cells were cultured for additional 48 hours to adhere, and then actin cytoskeleton was visualized. Shown are mean values from 3 repeats of assay ( \pm S.D.).

of the number of TUNEL-positive cells; a statistically significant increase (about $20 \%$ of positive cells) was only observed 6 hours after irradiation with 8 Gy (however, this could reflect residual radiation-induced DSBs). We concluded that isolated CECs showed apparent resistance to radiation-induced apoptosis in in vitro culture conditions.

\section{Radiation-induced actin stress fibres}

We previously analyzed radiation-related changes induced in vitro in the actin cytoskeleton of established human endothelial cell lines DMEC and HUVEC (Gabrys et al., 2007). Here we applied staining with fluorescentlylabelled phalloidin to detect radiation-induced changes in the structure of actin filaments in primary cardiac endothelial cells. CECs isolated from juvenile animals were irradiated in vitro with 2 or $8 \mathrm{~Gy}$, and then fixed and stained with phalloidin 6, 24 and 48 hours after irradiation. Cells that stained diffusely with phalloidin and cells that contained different numbers of contractile stress fibres could be distinguished. Figure 3A shows examples of cells differing in the number of such stress fibres. Figure $3 \mathrm{~B}$ shows that about half of non-irradiated CECs contained no stress fibres, while about $20 \%$ contained a high number of such fibres. Irradiation in vitro

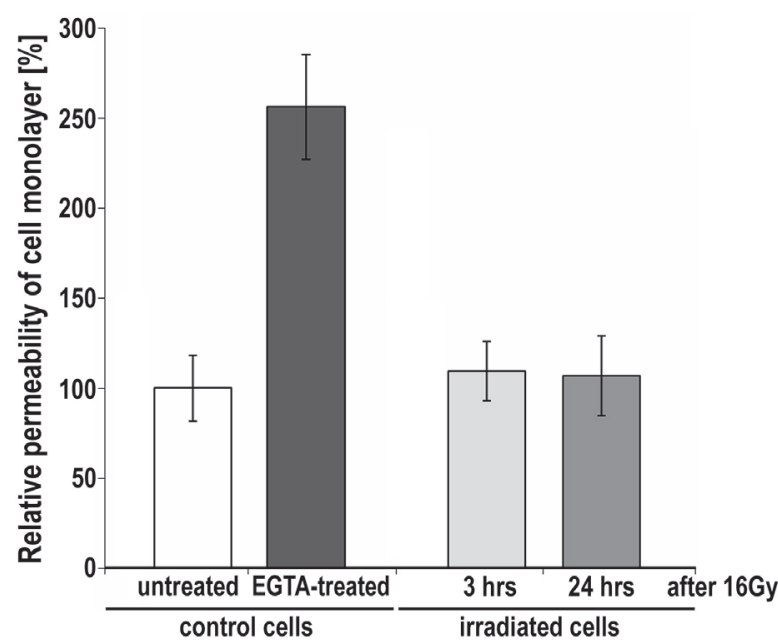

Figure 4. Effect of radiation on permeability of monolayer of cardiac endothelial cells

CECs isolated from juvenile mice were grown to form a monolayer, irradiated with $16 \mathrm{~Gy}$, incubated for additional 3 or 24 hours, and then permeability to FITC-dextran was assessed. Cells pre-incubated with $5 \mathrm{mM}$ EGTA were used as a positive control. Shown are relative values with average permeability of untreated controls set at $100 \%$ (mean values \pm S.D. from 3 independent experiments)

with 2 Gy resulted in some decrease in the proportion of fibre-free cells and an increase of those with a high content of stress fibres, yet those changes were only minor. Significant changes were observed in CECs irradiated in vitro with $8 \mathrm{~Gy}$. The fraction of cells with no stress fibres dropped to 30-35\% while that of cells with numerous fibres increased twofold (to about $40 \%$ ). In a second experiment we tested for radiation-induced stress fibres in cells irradiated in vivo. Mice were irradiated with 2 or $8 \mathrm{~Gy}$, and at 12 hours, 36 hours and 6 days after irradiation CECs were extracted. Isolated cells were cultured for an additional 48 hours to adhere, and then actin cytoskeletal structures were stained with phalloidin. Figure 3C shows that about half of CECs isolated from 8 weeks old control mice did not contain stress fibres, while only one fourth contained a high number of such fibres. Irradiation with $2 \mathrm{~Gy}$ increased the number of cells with a high number of stress fibres (to about $40 \%$ ) and reduced the number of stress fibre-free cells; such changes persisted for 6 days after the exposure. Irradiation with the higher dose (8 Gy) resulted in a highly significant increase in the abundance of stress fibres (more than $60 \%$ of cells contained numerous fibres 12 hours after irradiation; some reduction of the abundance of stress fibres could be observed at later times). We concluded that radiation induced dose-dependent changes in the actin cytoskeleton (i.e., formation of stress fibres) in cardiac endothelial cells irradiated in vivo or in vitro (notably, these effects appeared more significant in CECs irradiated in vivo in the context of a heart tissue).

\section{Radiation-induced changes in permeability of cell monolayer}

We showed previously that high doses of ionizing radiation (20 Gy) increased the permeability of DMEC monolayers to FITC-labelled dextran (Gabrys et al., 2007). Here we used the same methodology to test the effect of ionizing radiation on the permeability of monolayers formed by primary CECs isolated from juvenile 


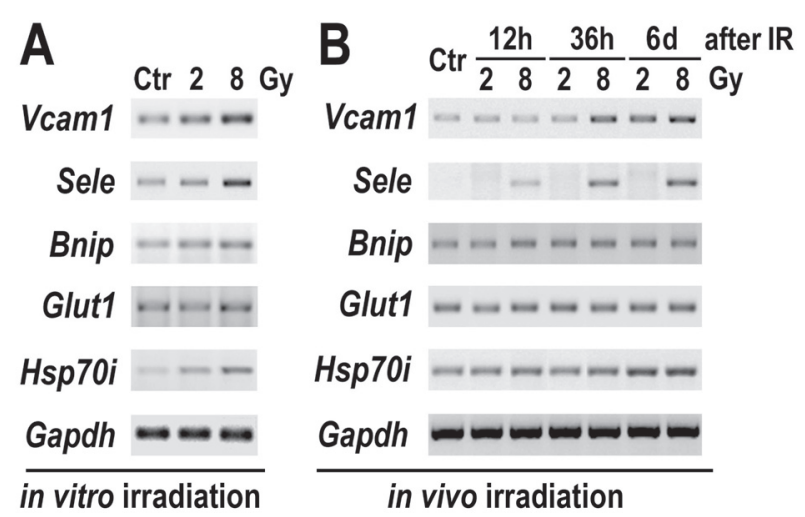

Figure 5. Radiation-induced changes in expression of selected genes

(A) CECs isolated from juvenile mice were irradiated in vitro with 2 or $8 \mathrm{~Gy}$, and RNA was isolated 24 hours after exposure. (B) Eightweek-old mice were irradiated in vivo with 2 or $8 \mathrm{~Gy}$, and CECs were isolated 12 and 36 hours, and 6 days after the exposure; RNA was purified directly after isolation of the cells.

mice. Figure 4 shows that irradiation in vitro with $16 \mathrm{~Gy}$ did not increase the permeability of monolayers formed by CECs to dextran 3 or 24 hours after exposure (although a significant increase in the number of stress fibres was observed in such cells; not shown). We concluded that even high doses of radiation failed to induce significant increase in the permeability of CEC's monolayer, suggesting resistance of the cell junctions formed between cells isolated from juvenile animals.

\section{Radiation-induced changes in gene expression}

Finally, to characterize the response of cardiac endothelial cells to ionizing radiation, changes in expression of selected genes were analyzed. CECs isolated from juvenile mice were irradiated in vitro with 2 or 8 Gy, and then 24 hours after the exposure RNA was purified. In addition, animals were irradiated in vivo with 2 or $8 \mathrm{~Gy}$, CECs were isolated 12 or 36 hours or 6 days after the exposure, and then RNA was purified directly after the cell isolation. Figure 5 presents the results of semi-quantitative RT-PCR analyses of the expression levels of V $\operatorname{cam} 1$ and Sele, two endothelium-specific inflammation-related genes previously reported to be affected by ionizing radiation (Heckmann et al., 1998; Nubel at al. 2004), Bnip3 and Glut1, two genes regulated by hypoxia (Guo et al., 2001; Airley et al., 2001), and Hsp70i, the major stress-inducible HSP gene (review in: Daugaard et al., 2007); Gapdh was used as a control housekeeping gene.
We observed up-regulation of V cam 1 gene in CECs irradiated in vitro at the higher dose. In addition, up-regulation of this gene was detected in CECs isolated 1.5 or 6 days after the exposure of animals to 8 Gy. Similarly, expression of Sele gene was up-regulated in CECs irradiated in vitro with $8 \mathrm{~Gy}$. Increased expression of this gene was also observed in CECs isolated $0.5,1.5$ or 6 days after the exposure of animals to the higher dose of radiation. Notably, we did not observe significant changes in the expression of several other genes putatively involved in response of endothelium to ionizing radiation: $C d h 5$, Igfbp3, Jun, Mmp9, Pai1 (not shown). Similarly, expression of the hypoxia-related Bnip and Glut1 genes was not affected in CECs exposed to ionizing radiation in vitro or in vivo. However, radiation caused up-regulation of $\mathrm{Hsp}_{s} 70 \mathrm{i}$, the major stress-inducible HSP, which was visible in a dose-dependent manner within 24 hours after irradiation in vitro. Interestingly, some up-regulation of $\mathrm{Hsp} 70 \mathrm{i}$ could also be detected in CECs isolated from irradiated animals 6 days after the exposure. We concluded that exposure of CECs to ionizing radiation in vitro or in vivo resulted in up-regulation of certain stress-related genes.

\section{DISCUSSION}

We described a procedure of immunoselection of cells optimized for isolation of mouse cardiac endothelial cells (mostly of microvascular origin). The procedure was used to purify cells from donors at a wide range of age (from newborns to elderly animals). We found that primary CECs isolated from newborn/juvenile mice were the most vital and could be cultured in vitro for at least 5-6 weeks, hence such cells seem the best option for medium-term in vitro experiments with cells irradiated in culture. This conclusion was consistent with previous reports, where endothelial cells isolated exclusively from juvenile mice were used for in vitro studies (Lim et al., 2003). In addition we found that CECs could be isolated from adult donors (up to 6-7 months of age), and that such cells could be cultured for 2-3 weeks being suitable for different types of in vitro experiments (e.g., for investigation of the structure of the cytoskeleton). Notably, this is the first time when isolation of viable CECs from adult 6 months old mice was described. Earlier papers reported isolation and culture of endothelial cells obtained from mice not older than 6-10 weeks (Dong et al., 1997; Marelli-Berg et al., 2000; Lim et al., 2003). Importantly, CECs could be isolated from hearts of adult animals after their irradiation in vivo, and thus our model becomes a new option for studying long-term effects of ionizing radiation. Moreover, CECs obtained from older

Table 1. Efficiency of isolation and culturing of cardiac endothelial cells isolated from hearts of mice of different age Number of total adherent cells: ${ }^{1}$ refers to number of cells after the end of first passage (one week after selection; this number also reflects the rate of proliferation). Number of total non-adherent cells: 2refers to combined number of cells washed out during three medium changes before the end of first passage. Shown are average values from 2-3 experiments with independent preparations of cells.

\begin{tabular}{|c|c|c|c|c|}
\hline \multirow{2}{*}{ Age of mice } & \multicolumn{3}{|c|}{ Average numbers of CECs per one heart: } & \multirow{2}{*}{$\begin{array}{l}\text { Minimal persistence of viable CECs in culture } \\
\text { (weeks) }\end{array}$} \\
\hline & directly after selection & total adherent ${ }^{1}$ & total non-adherent ${ }^{2}$ & \\
\hline $1-3$ days & 120000 & 190000 & 40000 & 5 \\
\hline 10 weeks & 270000 & 130000 & 190000 & 3 \\
\hline 20 weeks & 260000 & 65000 & 220000 & 2 \\
\hline 28 weeks & 210000 & 60000 & 180000 & 2 \\
\hline 48 weeks & 240000 & 0 & $\sim 240000$ & - \\
\hline 88 weeks & 300000 & 0 & $\sim 300000$ & - \\
\hline
\end{tabular}


mice (10 months and older) have lost their ability to adhere and grow in vitro in the culture dish; however, such cells could be used for experiments immediately after their purification (e.g., for gene expression studies).

Endothelial cells from cardiac vasculature are a potential target for radiation-induced changes involved in cardiovascular disease (Fajardo \& Stewart, 1971). Here we used endothelial cells isolated from hearts of irradiated animals, as well as cultured cells exposed to radiation in vitro, as models for testing parameters potentially involved in the development of cardiovascular system malfunctions. These parameters included radiation-induced changes in the structure of the cytoskeleton and cell junctions, and in gene expression. The integrity of the endothelium is controlled by the stability of cellular junctions and tensile forces maintained by the actin cytoskeleton (Kantak et al., 1993; Waters et al., 1996). We described previously that endothelial cells from skin microvasculature (but not from a large vessel) responded to radiation in vitro by a rapid and dose-dependent formation of so called actin stress fibres (Gabrys et al., 2007). Here we found that irradiation of cardiac endothelial cells also resulted in the formation of actin stress fibres. Interestingly, the observed changes seemed more evident in cells irradiated in vivo, in the context of intact tissue. Formation of stress fibres affects cell motility, contractile responses and cell-to-cell junctions, thus could be involved in changes of the endothelial barrier functioning in irradiated animals. Radiation-induced changes in the structure of the cytoskeleton and cell junctions could result in the formation of gaps and increased permeability of monolayers formed by endothelial cells (Onoda et al., 1999). We already showed that high doses of ionizing radiation $(20 \mathrm{~Gy})$ increased the permeability of DMEC but not HUVEC monolayers (Gabrys et al., 2007). We found here that high doses of radiation $(16 \mathrm{~Gy})$ failed to induce a significant increase in the permeability of a monolayer formed in vitro by cardiac endothelial cells isolated from juvenile animals. However, the effects of ionizing radiation on the barrier function of the endothelium in the irradiated heart remain to be established. Several studies reported that endothelial cells responded to irradiation by elevated expression of pro-inflammatory molecules, which enhanced adhesion of leukocytes and their migration through the endothelium (Hallahan et al., 1996; Tsujino et al., 1999). We found increased expression of two such adhesion molecules, Vcam1 and Sel-E, in irradiated cardiac endothelial cells. In addition, an increased expression of the major stress-inducible heat shock protein Hsp70i was detected in such cells. The changes observed in irradiated cells, i.e., increased formation of actin stress fibres and increased expression of stress-response genes, yet their possible resistance to apoptosis, suggest cytostatic effects of radiation upon cardiac endothelial cells with a potential contribution to systemic cardiotoxicity.

\section{Acknowledgements}

The research received funding from the European Atomic Energy Community's Seventh Framework Programme (FP7/2007-2011) under grant agreement no. 211403 (Cardiorisk).

\section{REFERENCES}

Airley R, Loncaster J, Davidson S, Bromley M, Roberts S, Patterson A, Hunter R, Stratford I, West C (2001) Glucose transporter Glut-1 expression correlates with tumor hypoxia and predicts metastasis- free survival in advanced carcinoma of the cervix. Clin Cancer Res 7: 928-934.

Celermajer DS (1997) Endothelial dysfunction: does it matter? Is it reversible? I Am Coll Cardiol 30: 325-333.

Darby SC, Cutter DJ, Boerma M, Constine LS, Fajaro LF, Kodama K, Mabuchi K, Marks LB, Mettler FA, Pierce LJ, Trott KR, Yeh ET, Shore RE (2010) Radiation-related heart disease: current knowledge and future prospects. Int J Radiat Oncol Biol Phys 76: 656-665.

Daugaard M, Rohde M, Jäättelä M (2007) The heat shock protein 70 family: highly homologous proteins with overlapping and distinct functions. FEBS Lett 581: 3702-3710.

Dimri GP, Lee X, Basile G, Acosta M, Scott G, Roskelley C, Medrano EE, Linskens M, Rubelj I, Pereira-Smith O, Peacocke M, Campisi J (1995) A biomarker that identifies senescent human cells in culture and in aging skin in vivo. Proc Natl Acad Sci USA 92: 9363-9367.

Dong QG, Bernasconi S, Lostaglio S, De Calmanovici RW, MartinPadura I, Breviario F, Garlanda C, Ramponi S, Mantovani A, Vecchi A (1997) A general strategy for isolation of endothelial cells from murine tissues; characterization of two endothelial cell lines from the murine lung and subcutaneous sponge implants. Arterioscler Thromb Vasc Biol 17: 1599-1604.

Fajardo LF, Stewart JR (1971) Capillary injury preceding radiation induced myocardial fibrosis. Radiology 101: 429-433.

Gabrys D, Greco O, Patel G, Prise KM, Tozer GM, Kanthou C (2007) Radiation effects on the cytoskeleton of endothelial cells and endothelial monolayer permeability. Int J Radiat Oncol Biol Phys 69: 1553-1562.

Guo K, Searfoss G, Krolikowski D, Pagnoni M, Franks C, Clark K, Yu KT, Jayea M, Ivashchenko Y (2001) Hypoxia induces the expression of the pro-apoptotic gene BNIP3. Cell Death Differ 8: 367376.

Hallahan D, Kuchibhotla J, Wyble C (1996) Cell adhesion molecules mediate radiation-induced leukocyte adhesion to the vascular endothelium. Cancer Res 56: 5150-5155.

Hancock SL, Tucker MA, Hoppe RT (1993) Factors affecting late mortality from heart disease after treatment for Hodgkin's disease. $J$ Am Med Assoc 270: 1949-1955.

Heckmann M, Douwes K, Peter R, Degitz K (1998) Vascular activation of adhesion molecule mRNA and cell surface expression by ionizing radiation. Exp Cell Res 238: 148-154.

Hendry JH, Akahoshi M, Wang LS, Lipshultz SE, Stewart FA, Trott KR (2008) Radiation-induced cardiovascular injury. Radiat Environ Biophys 47: 189-193.

Hinsbergh VV (1997) Endothelial permeability for macromolecules. Mechanistic aspects of pathophysiological modulation. Arterioscler Thromb Vasc Biol 17: 1018-1023.

Kantak SS, Diglio CA, Onoda JM (1993) Low dose radiation-induced endothelial cell retraction. Int J Radiat Biol 64: 319-328.

Lim Y, Garcia-Cardena G, Allport JR, Zervoglos M, Connolly AJ, Gimbrone MA, Luscinskas FW (2003) Heterogeneity of endothelial cells from different organ sites in T-cell subset recruitment. Am J Pathol 162: 1591-1601.

Little MP, Tawn EJ, Tzoulaki I, Wakeford R, Hildebrandt G, Paris F, Tapio S, Elliotta P (2008) A systematic review of epidemiological associations between low and moderate doses of ionizing radiation and late cardiovascular effects, and their possible mechanisms. $\mathrm{R} a$ diat Res 169: 99-109.

Marelli-Berg FM, Peek E, Lidington EA, Stauss HJ, Lechler RI (2000) Isolation of endothelial cells from murine tissue. J Immunol Methods 244: 205-215.

Nubel T, Dippold W, Kaina B, Fritz G (2004) Ionizing radiation-induced E-selectin gene expression and tumor cell adhesion is inhibited by lovastatin and all-trans retinoic acid. Carcinogenesis 25: 13351344.

Onoda J, Kantak S, Diglio C (1999) Radiation induced endothelial cell retraction in vitro: correlation with acute pulmonary edema. Path Oncol Res 5: 49-55.

Petty RG, Pearson JD (1989) Endothelium - the axis of vascular health and disease. J R Coll Phys 23: 92-101.

Ponnuchamy B, Khalil RA (2008) Role of ADAMs in endothelial cell permeability: cadherin shedding and leukocyte rolling. Circ Res 102: 1139-1142.

Rogakou EP, Pilch DR, Orr AH, Ivanova VS, Bonner WM (1998) DNA double-stranded breaks induce histone H2AX phosphorylation on serine 139. J Biol Chem 273: 5858-5868.

Schultz-Hector S, Trott KR (2007) Radiation-induced cardiovascular diseases: is the epidemiologic evidence compatible with the radiobiologic data? Int J Radiat Oncol Biol Phys 67: 10-18.

Senkus-Konefka E, Jassem J (2007) Cardiovascular effects of breast cancer radiotherapy. Cancer Treat Rev 33: 578-593.

Shimizu Y, Kodama K, Nishi N, Kasagi F, Suyama A, Soda M, Grant EJ, Sugiyama H, Sakata R, Moriwaki H, Hayashi M, Konda M, Shore RE (2010) Radiation exposure and circulatory disease risk: Hiroshima and Nagasaki atomic bomb survivor data, 1950-2003. BMJ 340: b5349. 
Tsujino K, Kodama A, Kanaoka N, Maruta T, Kono M (1999) Expression of pulmonary mRNA encoding ICAM-1, VCAM-1, and P-selectin following thoracic irradiation in mice. Radiat Med 4: 283-287.

Ward JF (1988) DNA damage produced by ionizing radiation in mammalian cells: identities, mechanisms of formation and repairability. Prog Nucleic Acid Res Mol Biol 35: 95-125.
Waters C, Taylor J, Moltenti A, Ward W (1996) Dose-response effects of radiation on the permeability of endothelial cells in culture. Cancer Res 46: 321-328. 